\title{
Neural Spike Feature Extraction of Globus Pallidus Based on Wavelet Package Decomposition
}

\author{
Yan $\mathrm{HE}^{\mathrm{a}}$, Jue WANG ${ }^{\mathrm{a}}$, Guangjun ZHANG ${ }^{\mathrm{a}}$, Guodong GAO ${ }^{\mathrm{b}, *}$ \\ ${ }^{a}$ The Key Laboratory of Biomedical Information Engineering of Ministry of Education, and Institute of \\ Biomedical Engineering, School of Life Science and Technology, Xi'an Jiaotong University, Xi'an 710049, \\ China \\ ${ }^{b}$ Department of Neurosurgery, Tangdu Hospital, Xi'an 710049, China
}

\begin{abstract}
In this study, features of neural signal recordings with microelectrode injected into the internal globus pallidus of non-human primates before and after 1-methyl-4-phenyl-1, 2, 3, 6-tetrahydropyridine (MPTP) treatment which induces Parkinson's disease (PD) were investigated. Neural oscillations were analyzed using the measure of wavelet spectrum and singular value extraction. Differences in the time-frequency analysis were observed between groups. Results showed that the PD states had significantly higher amplitudes of spectrum. Singular values extracted from wavelet coefficients after wavelet package decomposition were used to differentiate pathological changes from normal states, and the singular values of the reconstructed wavelet coefficients of the PD states were statistically smaller than controls. We speculate that the irregular high frequency oscillations and reduced singular values observed in the pathological firings are consistent with the previous view that a loss of information transmission in the neural circuitry underlies PD and these measurements could be of diagnostic and monitoring values of the disease state.
\end{abstract}

Index Terms: Parkinson's Disease; Neuronal Oscillation; Wavelet Packet Decomposition; Singular Value

(C) 2011 Published by MECS Publisher. Selection and/or peer review under responsibility of the Research Association of Modern Education and Computer Science.

\section{Introduction}

The pathophysiology of the PD disease is related with the loss of pigmented dopaminergic neurons which leads to alterations in the activity of the neural circuits within the basal ganglia that regulate movement [1-2]. The role of dopamine within the basal ganglia appears to be complex [3] while PD is an age-related neurological disorder viewed as a dysfunction of information processing or disruption of the dynamics of neural information processing in the neural circuits in the basal ganglia. The information processing in the Parkinsonism basal ganglia involves not only activities of individual rhythms, but also interactions between rhythms.

* Corresponding author:

E-mail address: taohuacun@gmail.com 
Many previous studies have reported changes in the neural activity of globus pallidus (GP) neurons in MPTP-treated monkeys compared with healthy ones while firing rates of the internal globus pallidus (GPi) cells are increased, and the external globus pallidus $(\mathrm{GPe})$ cells display decreased firing rates in PD subjects.

Another example is the low frequency modulation of firing activity that arises in PD[4]. The cross-correlation analysis of simultaneously recorded pallidal cells in MPTP-treated monkeys revealed that the spiking activities of many of these neurons are synchronized [5]. Mean discharge rates, instantaneous discharge rates, interspike interval (ISI) histograms, and autocorrelation have all been used to assess spontaneous neuronal activities. However, due to the complexity and large amounts of data, the previous studies are unable to uncover more dynamical changes hidden in the spike train, more effective neuronal spike train decoding and feature extraction methods are needed for delineation and characterization of these changes in discharge neural signal. In this paper, we will mainly focus on the research of feature extraction of neuronal oscillatory activity of Gpi before and after incidence of the disease determined by the MPTP intervention in the animal model and try to capture the dominant dynamic changes of the neuronal firing patterns under PD state.

\section{Material and Methods}

Wavelet decomposition can be realized by the Mallat Algorithm [6-7]. Wavelet packet decomposition (WPD) [8-9], sometimes known as just wavelet packets is a wavelet transform where the signal is passed through more filters than the digital wavelet transform, and it's an advantageous and favorable method in digital signal processing especially on the non-stationary signal such as neuronal spike trains which composed of different frequencies simultaneously. The capability of this mathematical microscope to analyze different scales of neural rhythms is proved to be a powerful tool for investigating multi-scale oscillations of the brain signals. Wavelet spectrum is adopted for the spectral representation of the neural signal analysis at first after signal de-noising by wavelet transform. The coefficients derived from the temporal spike profiles could be taken as feature extraction parameters [10-11]. For $n$ levels of decomposition the WPD produces $2 n(n=1,2,3 \ldots)$ different sets of coefficients as opposed to $3 n+1$ set for the discrete wavelet transform. In the theory of Singular value decomposition, any $m \times n$ real valued matrix A of rank $r$, where $r \leqq \min (m, n)$, can be decomposed as $\mathrm{A}=\mathrm{U \Sigma VT}$, where $\mathrm{U}$ is an $\mathrm{m} \times \mathrm{n}$ matrix with $\mathrm{UTU}=\mathrm{I}, \mathrm{V}$ is an orthogonal $\mathrm{n} \times \mathrm{n}$ matrix, and is an $\mathrm{n} \times \mathrm{n}$ diagonal matrix. The diagonal elements $\alpha_{1}, \alpha_{2} \ldots \alpha_{\mathrm{n}}$ of $\Sigma$ can be arranged in a non-increasing order and are called the singular values of $\mathrm{A}(\mathrm{SV})$.

The energy of special sub-bands and corresponding coefficients of wavelet packet

decomposition especially the largest singular values are selected as features which have maximal discriminability according to the Fisher distance criterion and can describe the main features of the processing signals based on the quantification of energy found in specific frequency bands at specific time locations during each spike profile.

The objective of feature extraction is to produce a suitable representation of neural signals for pathology recognition. We adopt one-dimensional case with a 3 level decomposition for feature extraction. The daubechies 4 (db4) wavelet [12] is chosen as the wavelet basis for neuron spike train analysis because of its adaptability to the spike shape by comparing the decomposition level required while keeping the energy as much as possible and the optimization criteria is set as entropy. Since that energy distribution of the original signal is related with the reconstructed decomposed wavelet coefficients, the projection coefficients have close biological relevance and would demonstrate statistical significant difference between signals.

\section{Results}

The MPTP induced monkey model of PD are produced the same way as it was described in [13]. Recording began as the stable neuronal firing came out and it would last for 300 s or longer with the electrode injected in the GPi [14]. 
We select two dominant kinds of representative neural spiking trains recorded from the primate model as follows: 1) rest spontaneous spike trains of GPi neuron of normal healthy monkey; 2) rest spontaneous spike trains of GPi neurons under pathological condition after MPTP intervention.

Fig. 1 and Fig. 2 demonstrate the normalized wavelet spectrum of the dominant neural signals. At one time spike trains are chosen according to their representation of the main characteristic of the data, and then the statistical result is taken as final measurement for further discussion.

As for the spectral analysis, the pathological neuron spike demonstrates significant irregular higher frequency oscillation compared with the normal ones. The decomposition of neural signals into transient oscillations could yield new knowledge about real signal components and shed light on neuronal mechanisms of information processing. As for WPD, its coefficients could has been taken as the measure to describe neural signals, which has an advantage that demonstrates signal's character by several coefficients, and the singular value of the matrix has two dominant features while one is its strong stability and the other is its inheritance of in the matrix reflecting underlying information.

Eight wavelet coefficients are selected as the basis matrix and Fig. 3 and Fig. 4 illustrates the largest singular values of WPD coefficients under the pathological and normal conditions, and the pathological ones are lower than that of the normal ones. More details are illustrated in Table 1. These irregular higher frequency oscillations are closely related with the loss of energy and the miscarriage of information which induce the symptoms of PD.
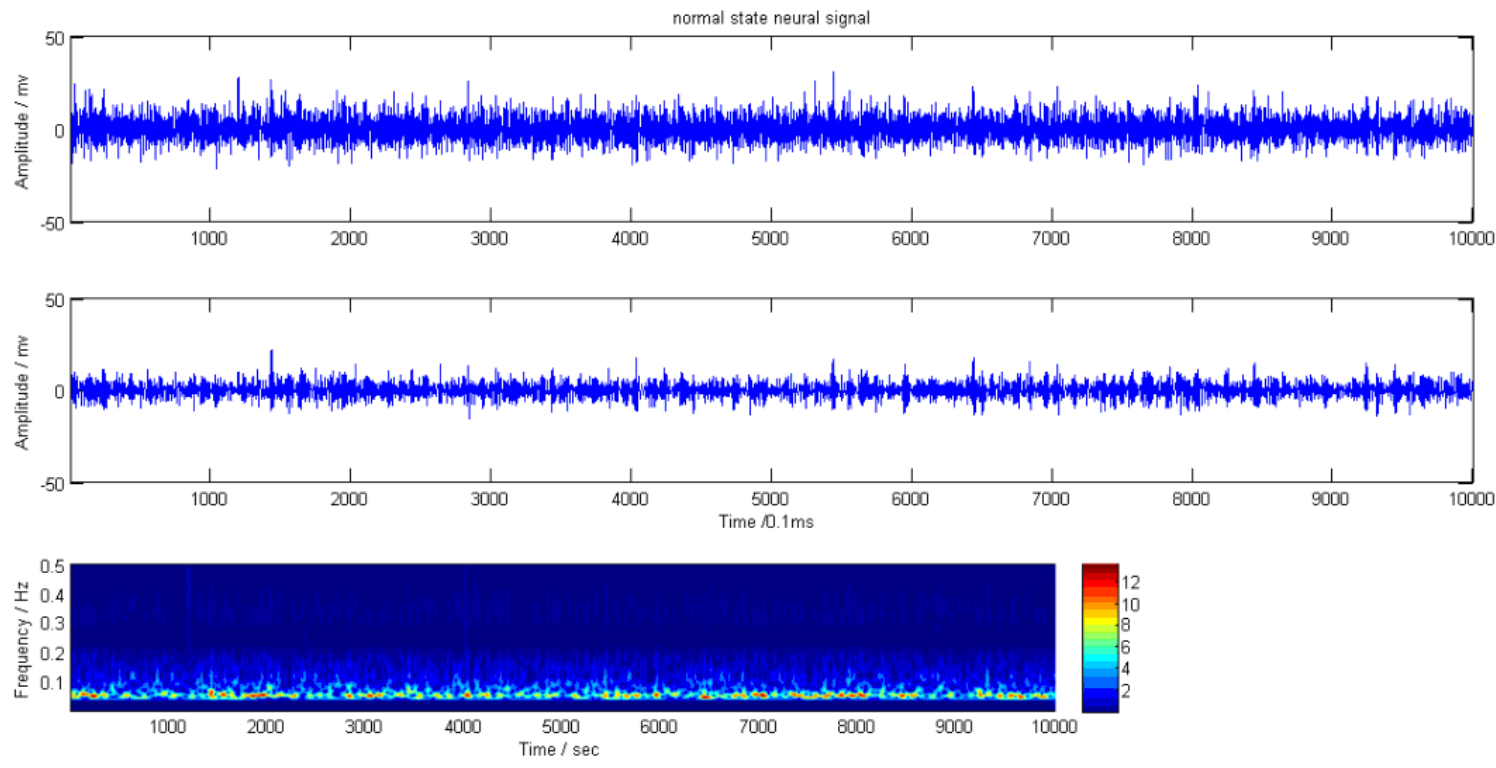

Fig. 1. Wavelet spectrum of neural signal under normal state 

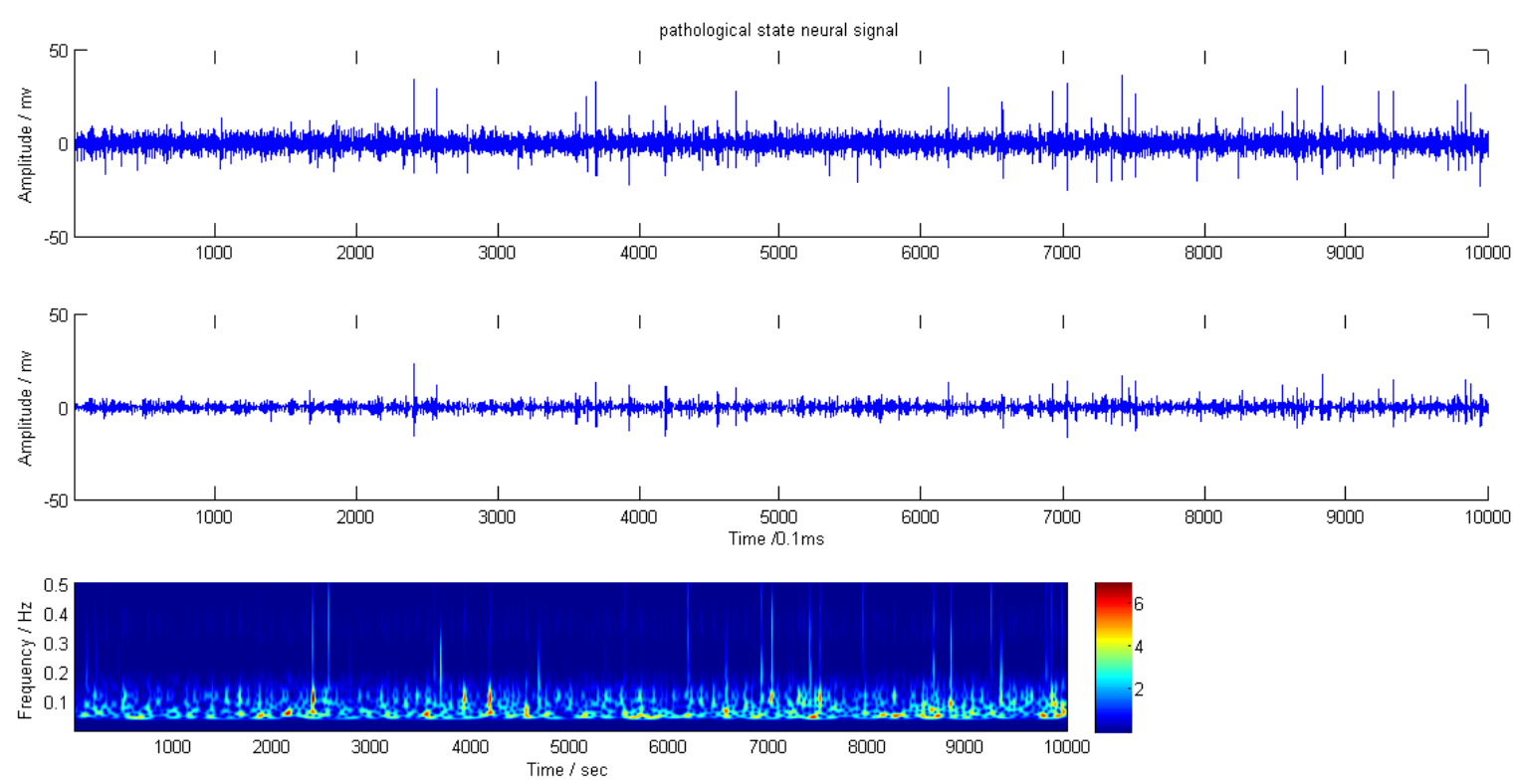

Fig. 2. Wavelet spectrum of neural signal under pathological state

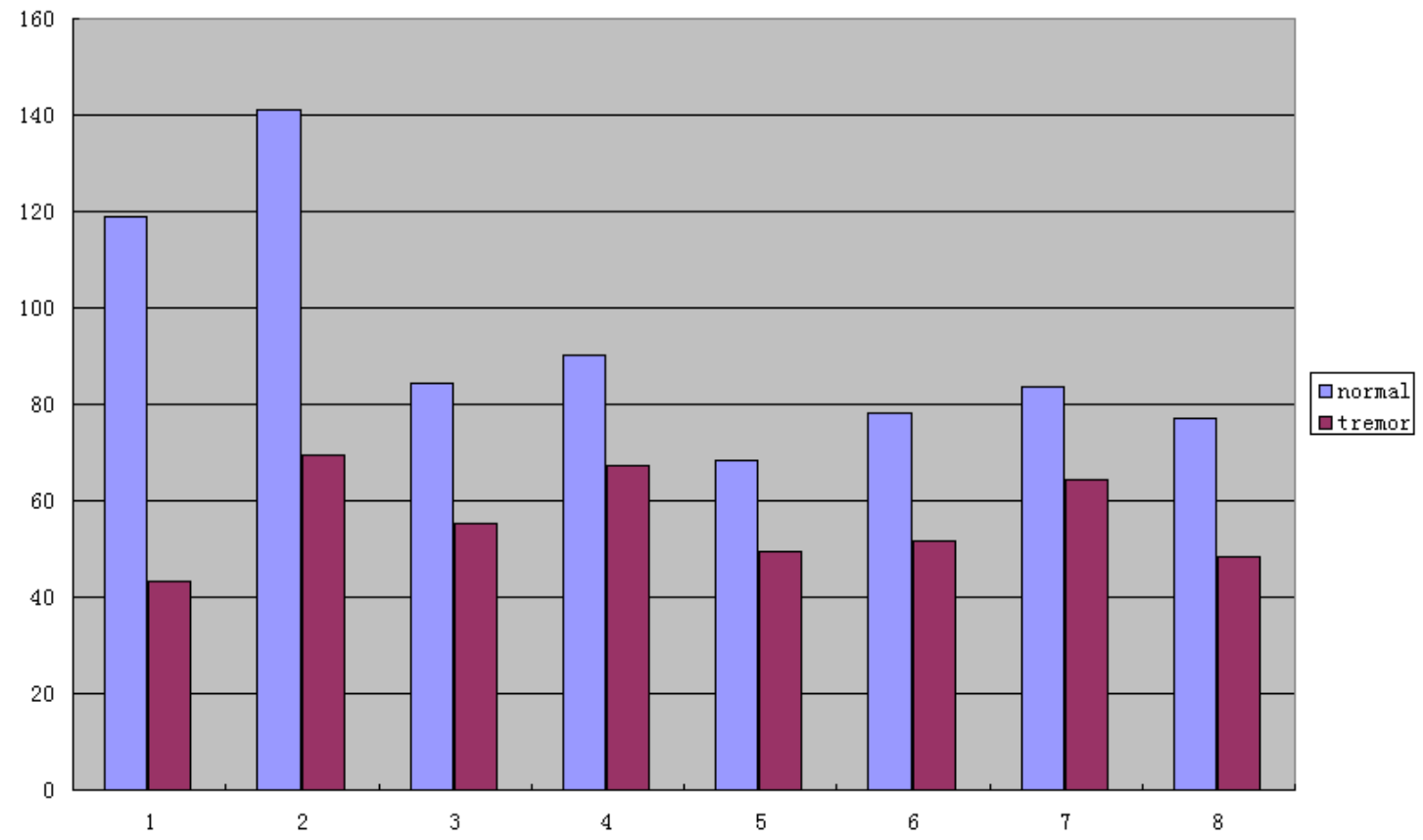

Fig. 3. The largest singular value of the eight level wavelet coefficients (d1-d8) of one sample 
singular value of $\mathrm{d} 1$

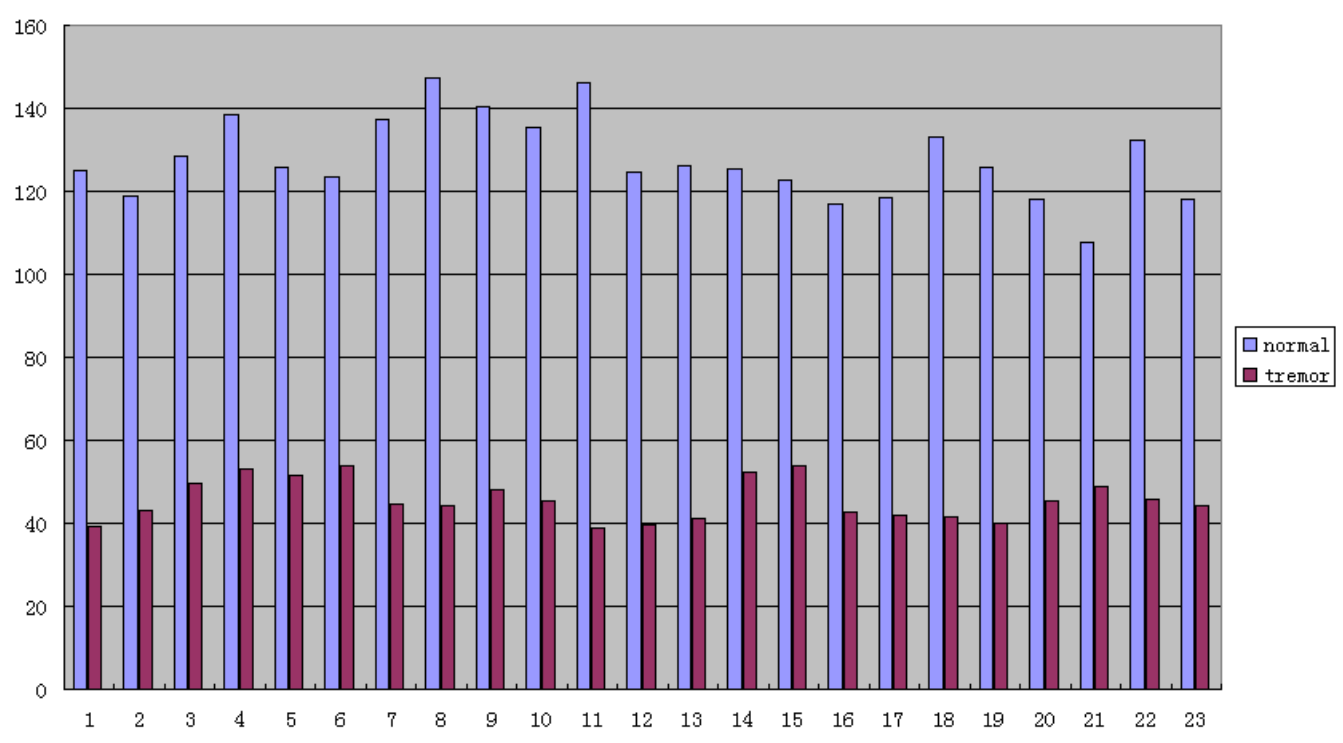

Fig. 4. The largest singular value of the first level wavelet coefficients (d1) of 23 Samples

Table 1. Paired-sample test for siingular values under pd state and normal state

\begin{tabular}{|c|c|c|c|c|c|c|c|c|}
\hline \multirow{3}{*}{ PD-Normal } & \multicolumn{5}{|c|}{ Paired differences } & \multirow[t]{3}{*}{ t } & \multirow{3}{*}{$\begin{array}{l}\text { Degree of } \\
\text { freedom }\end{array}$} & \multirow[t]{3}{*}{ Sig. } \\
\hline & \multirow[t]{2}{*}{ Mean } & \multirow[t]{2}{*}{$\begin{array}{c}\text { Std. } \\
\text { deviation }\end{array}$} & \multirow[t]{2}{*}{$\begin{array}{c}\text { Std. error } \\
\text { mean }\end{array}$} & \multicolumn{2}{|c|}{$\begin{array}{l}95 \% \text { Confidence interval } \\
\text { of the difference }\end{array}$} & & & \\
\hline & & & & Lower & Upper & & & \\
\hline D1 & 76.04763 & 12.93881 & 2.69793 & 70.45247 & 81.64280 & 28.187 & 22 & .000 \\
\hline D2 & $\mathbf{5 7 . 7 4 3 6 8}$ & 24.27094 & 5.06084 & 44.24813 & 65.23922 & 10.817 & 22 & .000 \\
\hline D3 & 17.15155 & 10.33453 & 2.15490 & 12.68257 & 21.62054 & 7.959 & 22 & .000 \\
\hline D4 & 9.59830 & 14.95797 & 3.11895 & 3.11895 & 16.06661 & 3.077 & 22 & .006 \\
\hline D5 & 27.86826 & 4.93179 & 1.02835 & 25.73559 & 30.00092 & 27.100 & 22 & .000 \\
\hline D6 & 27.68020 & 5.65622 & 1.17940 & 25.23427 & 30.12613 & 23.470 & 22 & .000 \\
\hline D7 & 21.22481 & 8.06033 & 1.68069 & 17.73927 & 24.71036 & 12.629 & 22 & .000 \\
\hline D8 & 23.73851 & 5.67070 & 1.18242 & 21.28631 & 26.19070 & 20.076 & 22 & .000 \\
\hline
\end{tabular}

\section{Conclusion}

Clarifying the relationship between intrinsic oscillations of neural signals and the pathological states seems to be efficacious by way of WPD, which is simple and easier for implementation although the mathematical 
principle is complex. It helps us effectively explore the progressive but fatal changes not only in the frequency domain but also in temporal aspect; these evidences might be a strong support for the prediction, diagnosis and the evaluation of clinical treatment.

The objective of feature extraction is to produce a suitable representation of neural signals for PD recognition; we deduce the singular value is the signature of the information loss and incidence of the disease which could be used as the parameter for further diagnosis and surgery evaluation.

\section{Acknowledgements}

We thank Prof. Guodong GAO and his group for their cooperation in the experiment.

This work was supported in part by the grant from the National Natural Science Foundation (Grant No.30670660, Grant No.10872156).

\section{References}

[1] McCormick D.A, Bal T, Sleep and arousal: thalamocortical mechanisms, Annual Review of Neuroscience 20 (1997) 185-215.

[2] Bar-Gad, H Bergman H, Stepping out of the box: information processing in the neural networks of the basal ganglia, CURRENT OPINION IN NEUROBIOLOGY 11 (2001) 689-695.

[3] David Williams, Marina TIjssen, Gerard van Bruggen, Dopamine-dependent changes in the functional connectivity between basal ganlia and cerebral cortex in humans, Brain 125 (2002) 1558-1569.

[4] S. Morrison, G. Kerr, K.M. Newell, P.A. Silburn, Differential time- and frequency-dependent structure of postural sway and finger tremor in Parkinson's disease, Neuroscience Letters 443 (2008) 123-128.

[5] Aoki F, Changes in power and coherence of brain activity in human sensorimotor cortex during performance of visuomotor tasks, Biosystems 63 (2001) 89-99.

[6] Mallat S, A theory for multiresolution signal decomposition: the wavelet representation, IEEE Pattern Analysis and Machine Intelligence 11 (1989) 674-693.

[7] Mallat S, A Wavelet Tour of Signal Processing, Academic Press; 2nd edition, 1999.

[8] Daubechies I, Ten lectures on wavelets, CBMS-NSF conference series in applied mathematics. SIAM Ed.1992

[9] Daubechies I, The wavelet transform of time-frequency localization and signal analysis, IEEE transactions on Information Theory 36 (1990) 961-1005.

[10] Donoghue J. P, Neural discharge and local field potential oscillations in primate motor cortex during voluntary movements, Journal of Neurophysiology 79 (1998) 159-173.

[11] Simona Mrakic-Sposta , Sara Marceglia, Marcello Egidi, Giorgio Carrabba, Extracellular spike microrecordings from the subthalamic area in Parkinson's disease, Journal of Clinical Neuroscience 15 (2008) 559-567.

[12] Zenadic Z, J.W. Burdick, Spike detection using continuous wavelet transform, IEEE Transactions on Biomedical Engineering 52 (2005) 74-87.

[13] J M. Hurtado, Charles M. Gray, Laszlo B. Tamas, Karen A. Sigvardt, Dynamics of tremor-related oscillations in the human globus pallidus: A single case study, PNAS 96 (1999) 1674-1679.

[14] Yan He, Jue Wang, Globus pallidus neuron spike time series prediction based on local-region multi-step forecasting model, 3rd International Conference on Intelligent System and Knowledge Engineering (2008) $224-229$. 